\title{
Effect of silencing the T-Box transcription factor TBX2 in prostate cancer PC3 and LNCaP cells
}

\author{
WEN-LIANG DU*, QIAN FANG*', YUE CHEN, JING-WEI TENG, \\ YONG-SHUANG XIAO, PING XIE, BO JIN and JUN-QI WANG \\ Department of Urology, The Affiliated Hospital of Xuzhou Medical University, Xuzhou, Jiangsu 221002, P.R. China
}

Received March 3, 2017; Accepted July 31, 2017

DOI: $10.3892 / \mathrm{mmr} .2017 .7361$

\begin{abstract}
T-Box (TBX)-2 is a member of the T-box gene family, which is aberrantly expressed in numerous types of malignant tumors, and has previously been demonstrated to be conducive to tumor progression by acting as a transcription factor. However, specific information regarding the expression and function of TBX2 in prostate cancer cells remains to be elucidated. The present study demonstrated that silencing of TBX2 by TBX2 small interfering RNA inhibited cell proliferation and promoted cell senescence. It was demonstrated that knockdown of TBX2 inhibited cell metastatic abilities by upregulating E-cadherin and downregulating $\mathrm{N}$-cadherin, Vimentin and fibronectin. In addition, the expression of TBX2 in prostate cancer tissues and tumor adjacent tissues was detected by immunohistochemistry. The results indicated that the expression rates of TBX2 were significantly increased in the cancerous tissues, compared with the healthy tumor adjacent tissue, and TBX2 increased staining was associated with the clinical stage and pathological grade. The findings of the present study therefore suggest that TBX2 expression is markedly increased in prostate cancer and TBX2 may act as a potential beneficial therapeutic target for the future treatment of prostate cancer.
\end{abstract}

\section{Introduction}

Prostate cancer is one of the most common frequent malignant tumor in urinary system. Its morbidity and mortality are higher in developing countries than those of developed countries. In spite of the decrease in mortality due to the improvements in early detection and treatments, it is still the

Correspondence to: Professor Jun-Qi Wang, Department of Urology, The Affiliated Hospital of Xuzhou Medical University, 99 West Huai-hai Road, Xuzhou, Jiangsu 221002, P.R. China

E-mail: dwlkb3355@163.com

${ }^{*}$ Contributed equally

Key words: T-Box-2, prostate cancer, epithelial-mesenchymal transition, E-cadherin, snail second leading cause of cancer death. The majority of patients are diagnosed with clinically localized cancer which can be treated effectively with radical prostatectomy, radiotherapy or androgen deprivation therapy. However, advanced androgen dependent prostate cancer patients, which were treated with androgen deprivation therapy, almost invariably progressed to castration-resistant prostate cancer stage with the poor prognosis and high mortality. Therefore, it's important to expedite the development of effective therapeutic targets for prostate cancer.

T-box gene family is a transcription factor that plays an important role in the regulation of embryonic development and organ formation. TBX2 gene is one of the members of T-BOX gene family. TBX2 gene is involved in the formation of a variety of tissues and organs as well, including the heart, lungs, kidney, limb buds, mammary glands, gastrulation and cranial structures. TBX2 have been shown to function as transcriptional repressors. Recent studies have found that TBX2 gene has abnormal expression in many kinds of malignant tumors, and is associated with the cell proliferation, apoptosis and cell invasion. In breast cancer, TBX2 displays high expression in a cohort of primary breast cancers. Increased TBX2 expression is associated with metastases, and tumors with altered TBX2 expression also show poor overall survival (1). Simultaneously, the study has demonstrated that TBX2 is a strong cell-autonomous inducer of the epithelial-mesenchymal transition (EMT), and in the malignant human breast carcinoma cell lines MDA-MB-435 and MDA-MB-157, down regulation of endogenous TBX2 expression leads to a restitution of epithelial characteristics with reciprocal loss of mesenchymal markers and then inhibition of capacity of tumor cell migration and invasion (2). In colorectal cancer, the results showed significant correlations between tumor TBX2 overexpression and pTstage, distant metastasis, advanced AJCC stage and relapse. These data suggested that up regulation of TBX2 expression might contribute to tumor invasion and metastasis (3). In human melanoma cells, the activity of endogenous TBX2 are critically required to maintain proliferation and suppress senescence (4). Similarly, the recent study indicated that in contrast to normal tissues, TBX2 was involved in the carcinogenesis of endometrial adenocarcinoma, and was also associated with lymph node metastasis. Therefore, it might be a candidate therapeutic target for treatment of endometrial adenocarcinoma (5). 
However, the role of TBX2 gene in prostate cancer cells remains largely unknown. In our study, to explore whether TBX2 participates in the development and progression of prostate cancer, small interfering RNA (siRNA) targeted on TBX2 were transfected to silence the TBX2 expression in prostate cancer PC3 and LNCaP cells, and then the changes of biological function after decrease in expression of TBX2 were examined.

\section{Materials and methods}

Cell cultures. The human prostate cancer cell lines PC3 and LNCaP were purchased from Shanghai Institute of Biochemistry and Cell Biology (Shanghai, China). Two kinds of Cells were both grown in RPMI-1640 (HyClone, Logan, UT, USA) medium supplemented with $10 \%$ fetal bovine serum (FBS; Invitrogen, Shanghai, China) and 1\% penicillin-streptomycin (Beyotime Biotech, Jiangsu, China). Cells were kept at $37^{\circ} \mathrm{C}$ in a humidified atmosphere with $5 \% \mathrm{CO}_{2}$.

Antibodies and reagents. TBX2 polyclonal antibodies were purchased from Bioworld Technology, Inc., St. Louis Park, MN, USA. Polyclonal mouse antibodies against E-cadherin, $\mathrm{N}$-cadherin, Vimentin and Fibronectin were purchased from BD Biosciences (Franklin Lakes, NJ, USA). Snail and Twist polyclonal rabbit antibodies were purchased from ABclonal Biotechnology Co., Ltd., Cambridge, MA, US. Mouse monoclonal antibody against Mdm2, p53, Bax, Akt, p-Akt, VEGF and polyclonal rabbit antibodies against $\mathrm{Bcl}-2$ and $\beta$-actin were all purchased from Santa Cruz Biotechnology, Inc., Santa Cruz, CA. The secondary antibodies including goat anti-rabbit $\mathrm{IgG}$ and goat anti-mouse IgG were obtained from Zhongshan Golden Bridge Biotechnology Co., Ltd., Beijing, China. The standard of dilution was referenced in accordance to the antibody specification. TBX2 siRNA and negative control siRNA were designed, synthesized by GenePharma (Suzhou, China). Cell proliferation was measured by Cell Counting Kit- 8 (CCK-8; Beyotime Institute of Biotechnology, Nantong, China). Cell migration and Cell invasion ability were tested using transwell chambers (Corning Incorporated, Corning, NY, USA). Cell apoptosis was assessed by Annexin V-FITC/propidium iodide (PI) Apoptosis Detection kit (KeyGen, Nanjing, China).

SiRNA and transfection. The cells were divided into two groups: Transfection of TBX2 silenced by TBX2 siRNA as the experimental interference (TBX2 siRNA) group and transfection of negative control siRNA as the negative control group. When PC3 and LNCaP Cells cultured regularly were grown at a density of $60-70 \%$, TBX2 siRNA and negative control siRNA were respectively transfected into cells with serum-free medium by SiLentFect ${ }^{\mathrm{TM}}$ Lipid Reagent according to each reagent instruction. Six hours later, serum-free medium containing SiLentFect ${ }^{\mathrm{TM}}$ Lipid Reagent was removed from petri dish. The medium was replaced with the complete medium, and continued to culture for $48 \mathrm{~h}$, cells were gathered for the next assays.

Western blot analysis. Two kinds of Prostate cancer cells were collected after transfection for $48 \mathrm{~h}$ and washed three times with phosphate-buffered saline (PBS). Then by using cell lysis buffer the proteins were extracted at $4^{\circ} \mathrm{C}$ at $15,000 \mathrm{~g}$ for $5 \mathrm{~min}$. The total protein concentration was determined by BCA protein assay kit (Beyotime Biotechnology). Equal amounts of protein were separated on $15 \%$ sodium dodecyl sulfate-polyacrylamide gelelectrophoresis (SDS-PAGE; Beyotime Biotechnology), and then transferred onto nitrocellulose filter membranes (Millipore, Billerica, MA, USA). After the membranes were blocked in 5\% non-fat milk for $2 \mathrm{~h}$ at room temperature, they were incubated with corresponding primary antibodies with following dilution and $\beta$-actin antibody as internal reference at $4^{\circ} \mathrm{C}$ overnight (TBX2 1:500 dilution; E-cadherin, $\mathrm{N}$-cadherin 1:2,000 dilution; Vimentin 1:5,000 dilution; Fibronectin 1:8,000 dilution; Snail, Twist 1:1,200 dilution; MDM2, Bcl-2, p53, Bax, 1:200 dilution; p-Akt, Akt 1:100 dilution; VEGF 1:200 dilution). After the completion of the previous step, the membranes were washed with washing buffer 3 times for $15 \mathrm{~min}$, and then incubated with secondary antibody ( $\beta$-actin 1:10,000 dilution) at room temperature. The membranes were washed with washing buffer adding Tween-20 3 times again $2 \mathrm{~h}$ later. Finally, the bound antibodies were visualized by an enhanced chemiluminescence (ECL) reagent (Tanon Science and Technology Co., Ltd., Shanghai, China) and analysed using ImageJ software. This assay was performed in triplicate.

Cell proliferation assay. Two kinds of prostate cancer cells selected in logarithmic growth phase were seeded into 96-well plates with $100 \mu \mathrm{l}$ medium and continued to grow at $37^{\circ} \mathrm{C}$. PC3 cells were incubated with $4 \times 10^{3}$ cells per well, and LNCaP cells were incubated with $5 \times 10^{3}$ cells per well. When the cells were cultured at 24, 48, and 72 h, $10 \mu$ l CCK-8 solutions were added into every well, followed by cultivation at $37^{\circ} \mathrm{C}$ for $2 \mathrm{~h}$. The optical density (OD) values were determined at $450 \mathrm{~nm}$ through an ELX-800 spectrometer reader (Bio-Tek Instruments, Inc., Winooski, VT, USA). All six wells were tested in each experimental group, and this assay was performed in triplicate.

Cell migration assay. Two kinds of prostate cancer cells were gathered $48 \mathrm{~h}$ after transfection and then plated into the upper chamber. PC3 cells were at a density of $5 \times 10^{4}$ in $200 \mu \mathrm{l}$ per well, and LNCaP cells were $10 \times 10^{4}$ per well. Meanwhile, $600 \mu \mathrm{l}$ RPMI-1640 medium including 10\% FBS was added into the lower chamber. After $24 \mathrm{~h}$ cultivation, the medium in the upper chamber was removed and the cells on the side of the membrane were carefully wiped with cotton swabs. The cells that had been located at the lower surface of membrane were fixed with paraformaldehyde for $30 \mathrm{~min}$, and then stained by crystal violet for $15 \mathrm{~min}$. After the completion of the above operations, the upper chamber was washed using PBS, and the residual cells on the side of the membrane were wiped again. At last, the cells migrating through the chamber were photographed and counted (x200 magnifications). The average number of migrated cells was determined by counting four random areas of every chamber. This assay was repeated three times.

Cell invasion assay. Before conducting this assay, every upper chamber was precoated with $40 \mu 1$ Matrigel (BD Biosciences) diluted by serum-free medium (1:7 dilution). The next day, two kinds of prostate cancer cells were gathered and seeded into 
the upper transwell chamber with $600 \mu 1$ RPMI-1640 medium containing $10 \%$ FBS. The remaining experimental procedure was the same as the migration assay. This assay too was repeated three times.

Cell apoptosis assay. After transfection with TBX2 siRNA and negative control siRNA for $48 \mathrm{~h}$, two kinds of prostate cancer cells were gathered, centrifuged, and resuspended in $500 \mu \mathrm{l}$ binding buffer. Annexin V-FITC (5 $\mu \mathrm{l})$ and PI (5 $\mu \mathrm{l})$ were added and incubated at room temperature in the dark for $15 \mathrm{~min}$, and then this assay was conducted within $1 \mathrm{~h}$ on a flow cytometry (BD Biosciences).

Vascular tube formation assay. The $80 \mu 1$ Matrigel was thawed and taken into 48-well plates evenly. After being incubated at $37^{\circ} \mathrm{C}$ for $1 \mathrm{~h}$, human umbilical vein endothelial cells (HUVECs) were resuspended using the supernatant containing TBX2 siRNA or negative control siRNA were transferred to the 48 -well plates with a density of $4 \times 10^{4}$ per well respectively, and then continued to be incubated for $6 \mathrm{~h}$ at $37^{\circ} \mathrm{C}$. The cells were photographed to observe tube formation. Tube formed was quantified by counting the number of tube formed in every well.

Patients and samples. Fifty-three archived formalin-fixed, paraffin-embedded prostate cancer tissue specimens and tumor adjacent tissue specimens were collected in The Affiliated Hospital of Xuzhou Medical University during 2011-2016, and all of these cases were confirmed by pathological examination. None of the patients received preoperative adjuvant chemotherapy or radiotherapy. The age of all patients ranged

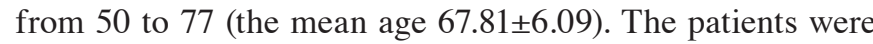
staged according to the 2012 American Association of Cancer (AJCC) TNM staging system: 6 were stage I, 27 were stage II, 17 were stage III, 3 were stage IV. Pathology was graded according to the Gleason grading system with the ISUP 2005 modification: Well differentiation (Gleason2-6): 19 cases; moderate differentiation (Gleason7): 23 cases; poor differentiation (Gleason8-10): 11 cases. Other histological cell types were not included in our study.

Immunohistochemistry. Immunoperoxidase tissue sections were dewaxed for $2 \mathrm{~h}$ at $65^{\circ} \mathrm{C}$, and then washed with xylene for $30 \mathrm{~min}$. The sections were blended in 100, 95, 85, 75 and 50\% ethanol in sequence. With the help of a microwave, citrate was heated to boiling, and then the sections were placed in it for $2 \mathrm{~min}$ at high heat and $8 \mathrm{~min}$ at medium heat. When the above operation was completed, citrate buffer needed to be cooled down to room temperature. Then after being washed with PBS for 15 min, hydrogen peroxide was added to the sections in order to block endogenous peroxidase activity. 30 min later, the sections were incubated with TBX2 polyclonal antibody (1:50 dilution) at $4{ }^{\circ} \mathrm{C}$ overnight after being washed with PBS again. The slices were rewarmed at room temperature, and then incubated with secondary antibody and streptavidin-peroxidase for 30 min successively. After being washed with PBS, the slices were stained by DAB, counterstained with hematoxylin, and mounted with neutral balsam. At last, the result of each section was evaluated by light microscopy. Evaluation of immunohistochemical staining: Staining intensity (0: no color; 1 : light yellow; 2 : brownish yellow; 3 : chocolate brown) and the percent of positive cells (0: $<10 \% ; 1: 11-30 \% ; 2: 30-60 \% ; 3:>60 \%)$, and the summation of the two gave the final score $(-: 0-1 ;+: 2-4$; ++ : $5-8 ;+++:$ 9).

Statistical analysis. The data were expressed as mean \pm standard deviation (SD). Statistical analyses were conducted via independent samples $t$ test using the software SPSS version 19.0 (SPSS, Chicago, USA). The relationship between TBX2 staining and clinicopathologic parameters of the patients was assessed by $\chi^{2}$ test and exact probability method. Every assay was carried out at least three times. $\mathrm{P}<0.05$ was considered to indicate a statistically significant difference.

\section{Results}

TBX2 siRNA decreases the expression of TBX2 in PC3 and LNCaP cells. The expression of TBX2 after transfection of TBX2 siRNA in PC3 and LNCaP cells was measured by western blot analysis. The results demonstrated that the protein expression of TBX2 was markedly reduced after silencing of TBX2 in prostate cancer cell lines ( $\mathrm{P}<0.01$; Fig. 1A).

Silencing of TBX2 represses the migration and invasion of prostate cancer cells. In order to evaluate the capacities of both two types of prostate cancer cells after knockdown of TBX2, Transwell migration and invasion assays were conducted. The migration results showed that the number of migrated cells in experimental interference (TBX2 siRNA) group were significantly reduced than that in the negative control group $(\mathrm{P}<0.01$; $\mathrm{P}<0.01$; Fig. 1B). Meanwhile, the invasion assay showed the similar results that the number of cells that penetrated through the matrigel-coated chambers in the TBX2 siRNA group were less than that in the negative control group $(\mathrm{P}<0.01 ; \mathrm{P}<0.01$; Fig. 1C). Based on the above data, we believed that silencing of TBX2 inhibited the abilities of migration and invasion of PC3 and $\mathrm{LNCaP}$ cell lines.

Downregulation of TBX2 inhibits the proliferation and promotes the apoptosis of prostate cancer cells. In order to evaluate the effect of TBX2 siRNA on cell growth, we performed the CCK- 8 assay. Compared with the negative control group, the ability of cell proliferation in TBX2 siRNA group was much weaker (Fig. 2A). The results suggested that silencing of TBX2 inhibited the proliferation of PC3 and LNCaP cells. Meanwhile, in order to determine whether the reduced proliferation was due to the increase of apoptosis, we carried out cell apoptosis assay by flow cytometry analysis. The assay results indicated that cells in TBX2 siRNA group had higher apoptosis rate in both two types of prostate cancer cells $(\mathrm{P}<0.01, \mathrm{P}<0.01$, Fig. $2 \mathrm{~B})$. Therefore, the above results suggested that knockdown of TBX2 promoted prostate cancer cell senescence.

Downregulation of TBX2 inhibits the tube formation of HUVECs. To evaluate effect of TBX2 siRNA on tube formation, we performed the vascular tube formation assay. Compared to the negative control siRNA, HUVECs with TBX2 siRNA showed a markedly reduction in the number of 
A

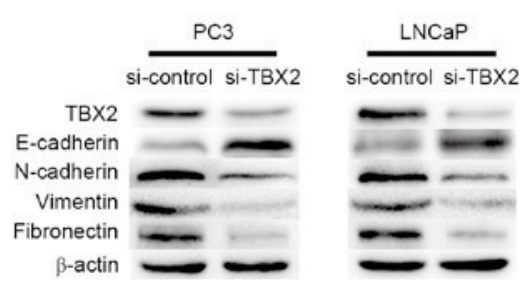

B

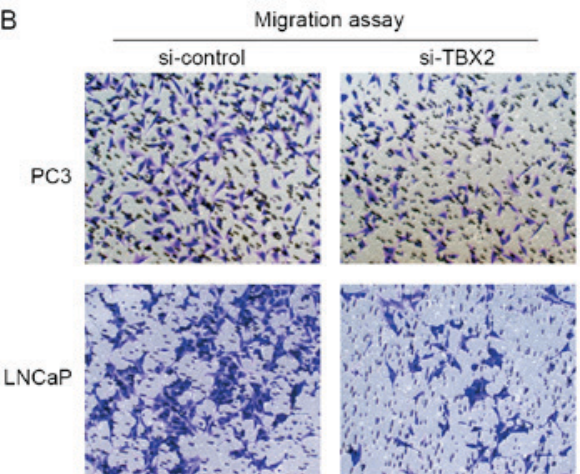

C

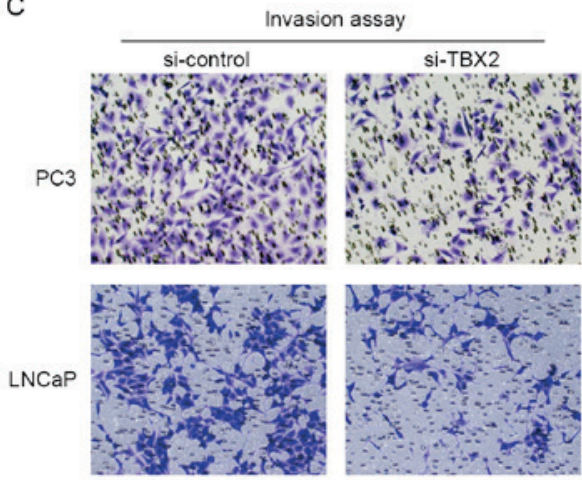

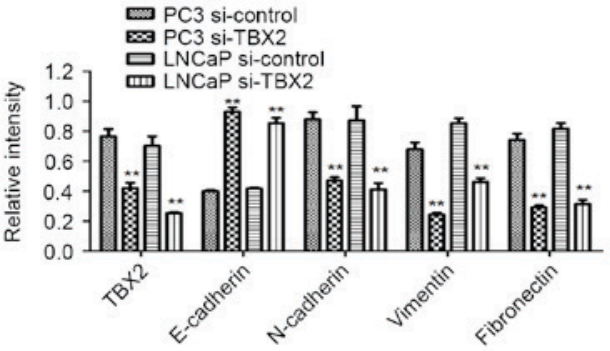
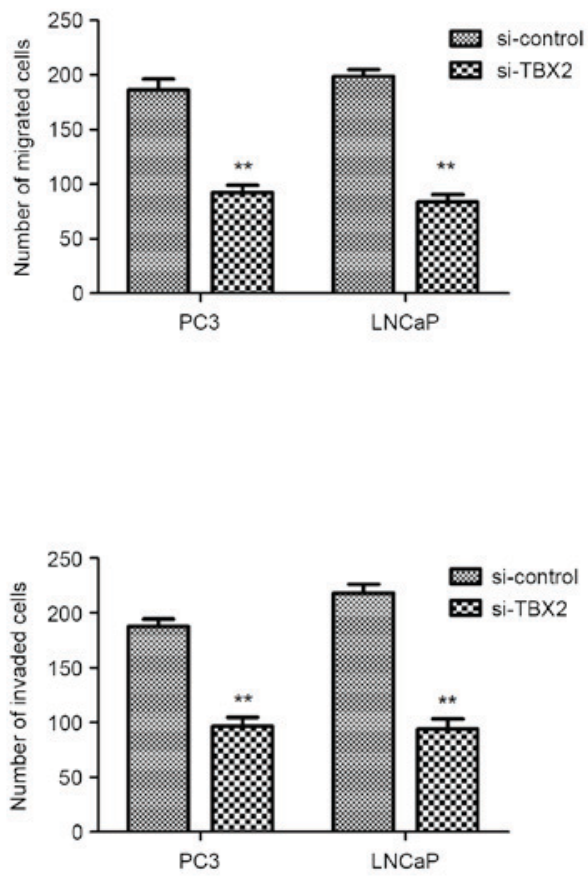

Figure 1. Silencing of TBX2 inhibits expression of TBX2 in PC3 and LNCaP cells, and also inhibits prostate cancer cell migration and invasion. (A) The protein level of TBX2, and EMT markers was detected by western blot analysis. $\beta$-actin was detected as an internal control. (B and C) Representative results showed that the PC3 and LNCaP cell migration and invasion in the TBX2 siRNA group were suppressed. All data were shown as mean \pm SD of triplicates ${ }^{* *} \mathrm{P}<0.01$.

tube formed $(\mathrm{P}<0.01, \mathrm{P}<0.01$, Fig. 3A). Then we used western blot analysis to determine the expression of VEGF in both two kinds of prostate cancer cells. The results showed that VEGF was decreased in TBX2 siRNA group, which indicated that low expression of VEGF, caused by silencing of TBX2 exerted an effect on endothelial cells ( $\mathrm{P}<0.01$; Fig. 3B).

Silencing of TBX2 blocks the process of EMT in part through downregulation of Snail/Twist in the prostate cancer cells. Based on fore-mentioned views, we performed the increasing studies, in which knockdown of TBX2 had effect on EMT that played a crucial role in tumor metastasis in prostate cancer cells. Western blot analysis showed that epithelial biomarker E-cadherin protein expression in the experimental interference (siRNA) group was significantly higher than that in the negative control group, while the mesenchymal biomarkers $\mathrm{N}$-cadherin, Vimentin and Fibronectin in TBX2 siRNA group were lower compared with that of the negative control group (Fig. 1A). the expression of EMT related transcription factors Snail and Twist in the experimental interference (TBX2 siRNA) group was significantly reduced compared with the negative control group. There were significant differences between TBX2 siRNA group and negative control group both in two types of prostate cancer cell lines (Fig. 4A).

Silencing of TBX2 impacts on the expression of the proteins related in PI3K/AKT signaling pathway. Increasing studies have demonstrated that PI3K/AKT signaling plays a critical function during cancer cell proliferation and cell apoptosis, so we detected the expressions of Akt, MDM2, p53, Bax and Bcl-2 via silencing of TBX2 in PC 3 and $\mathrm{LNCaP}$ cells. Western blot analysis showed that TBX2 knockdown led to the lower expression of p-Akt MDM2 and Bcl-2, while the increase of p53 and Bax. however, There was no significant difference in protein level of Akt between the two groups (Fig. 2C). These findings revealed that TBX2 might be involved in cell proliferation and apoptosis in prostate cancer cells.

Expression of TBX2 in prostate cancer tissues and correlations of clinical stage and pathological grade in prostate cancer. TBX2 was located in the cytoplasm of prostate cancer cells (Fig. 5). The expression rates of TBX2 were 
A
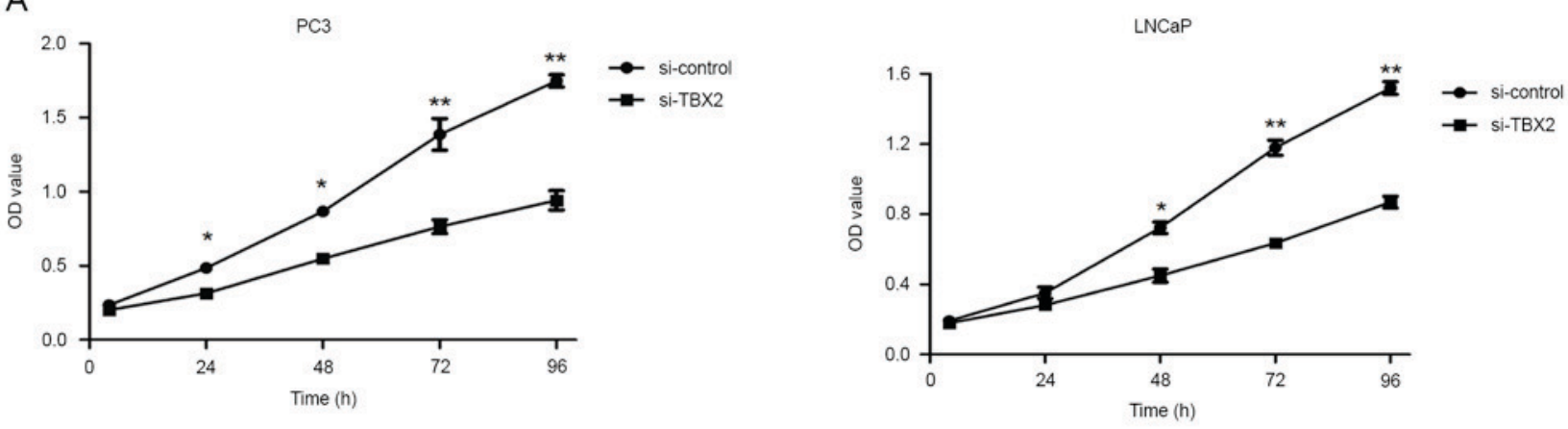

B
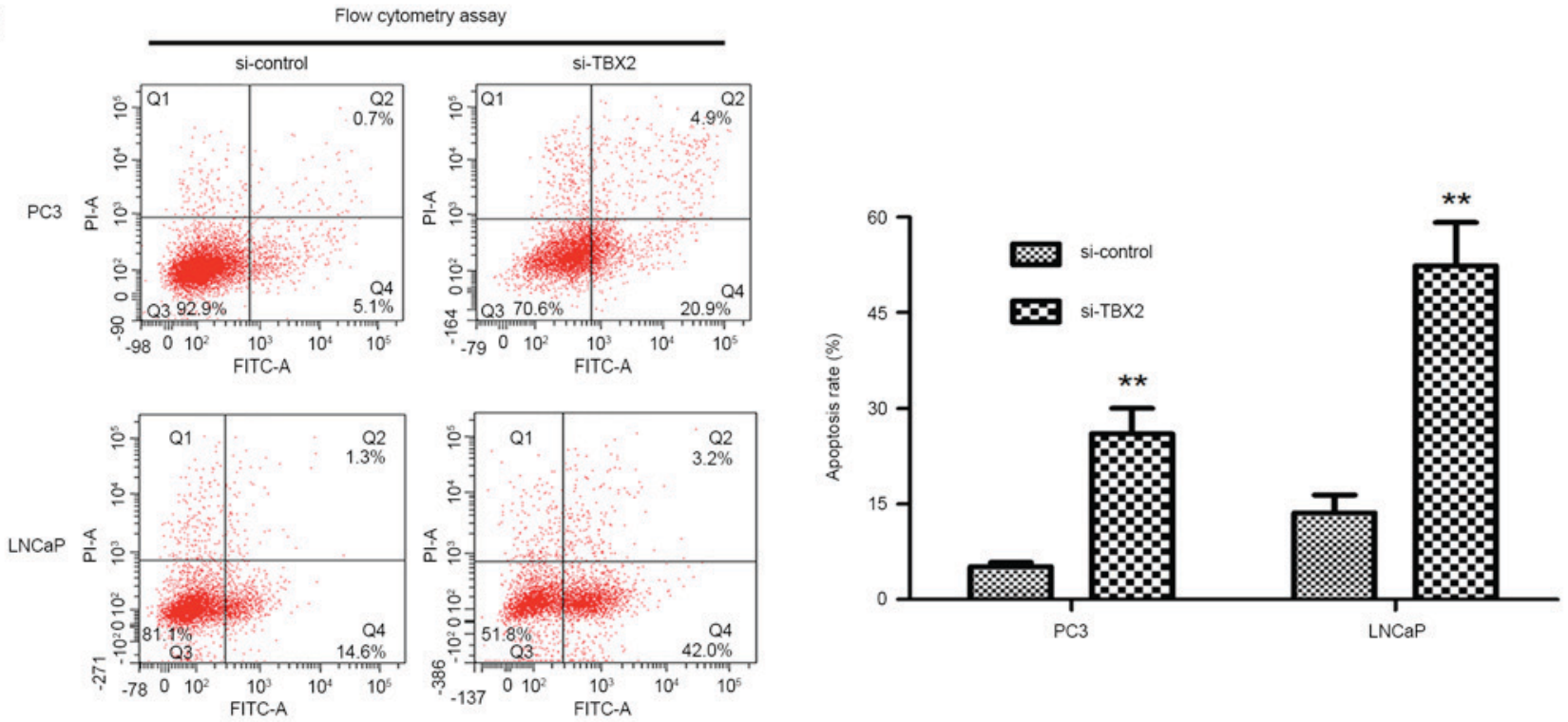

C
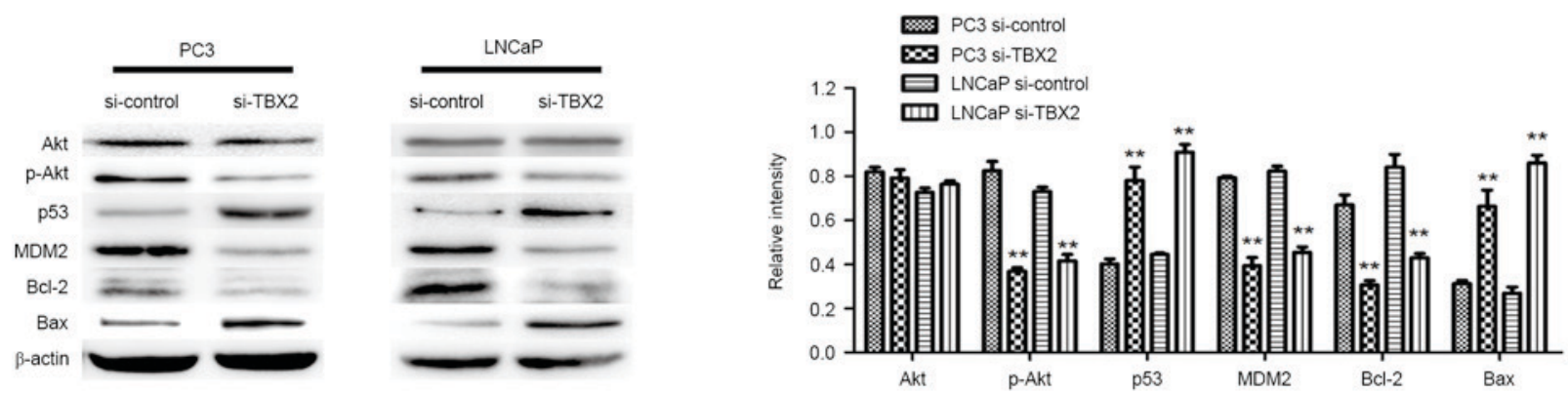

Figure 2. Silencing of TBX2 inhibits prostate cancer cell proliferation and promotes cell apoptosis. (A) The PC3 and LNCaP cells transfected by TBX2 siRNA and negative control siRNA respectively were incubated for $24 \mathrm{~h}$, and then were seeded in 96 -well plates with $100 \mu l$ medium. CCK- 8 reagents were added into every well, and the absorbance at $450 \mathrm{~nm}$ was measured. Cell proliferation of both two prostate cancer cells was markedly inhibited in the TBX2 siRNA group. (B) Cell apoptosis assay was performed by flow cytometry adding Annexin V-FITC/PI. Q2 region and Q4 region represent apoptotic cells, while Q3 region represents normal cells. The results showed that the apoptotic rate was increased in the TBX2 siRNA group. (C) The expressions of the proteins related in PI3K/AKT signaling pathway, including Akt, p-Akt, Bax, Bcl-2, p53 and MDM2, were measured by western blot analysis. All data were shown as mean \pm SD of triplicates. ${ }^{*} \mathrm{P}<0.05 ;{ }^{* * *} \mathrm{P}<0.01$.

$75.47 \%(40 / 53)$ in prostate cancer tissue, and were significantly higher than these of tumor adjacent tissue. A valuable difference in TBX2 positive staining was discovered between prostate cancer tissue and tumor adjacent tissue (Table I). Investigation of the relationship between TBX2 expression and clinicopathologic parameters were summarized in Table II. In prostate cancer, patients with poor differentiation showed more frequent expression of TBX2 than those with well and moderate differentiation. Meanwhile, TBX2 expression was positively correlated with clinical stage. However, TBX2 had no association with age and PSA (Table II).

\section{Discussion}

As one of the members of T-box gene family, TBX2 has been shown to play a critical role in embryonic development and organogenesis according to previous studies. Due to the overexpression of TBX2 in embryonic development, it can 
A
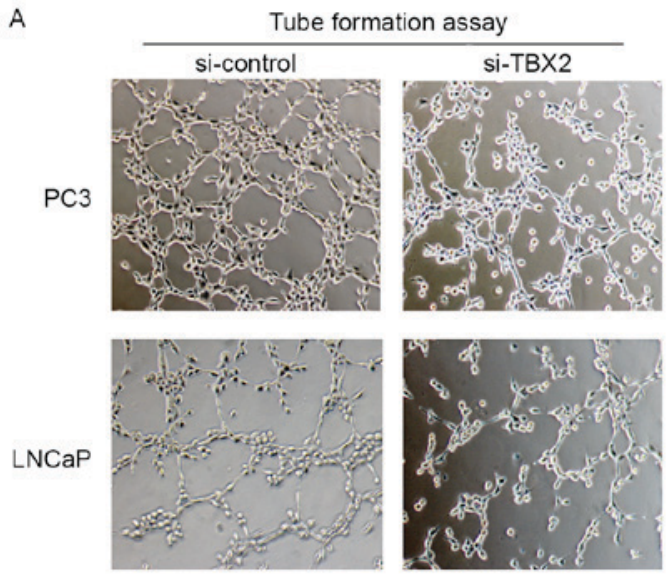

B

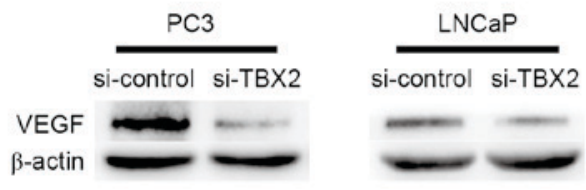

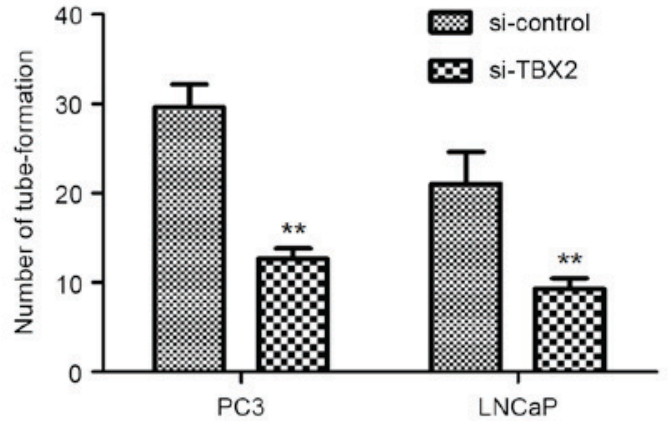

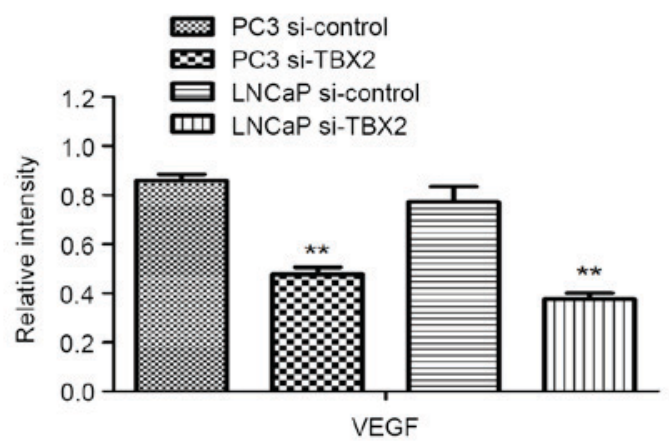

Figure 3. Silencing of TBX2 inhibits tube formation of HUVECs. (A) HUVEC tube formation assay was measured by the number of tube formed. The results showed that HUVECs with TBX2 siRNA were a markedly reduction in the number of tube formed compared with the negative control group. (B) The protein level of VEGF was determined by western blot analysis. $\beta$-actin was detected as an internal control. All data were shown as mean $\pm \mathrm{SD}$ of triplicates. ${ }^{*} \mathrm{P}<0.05$; ${ }^{* * *} \mathrm{P}<0.01$.
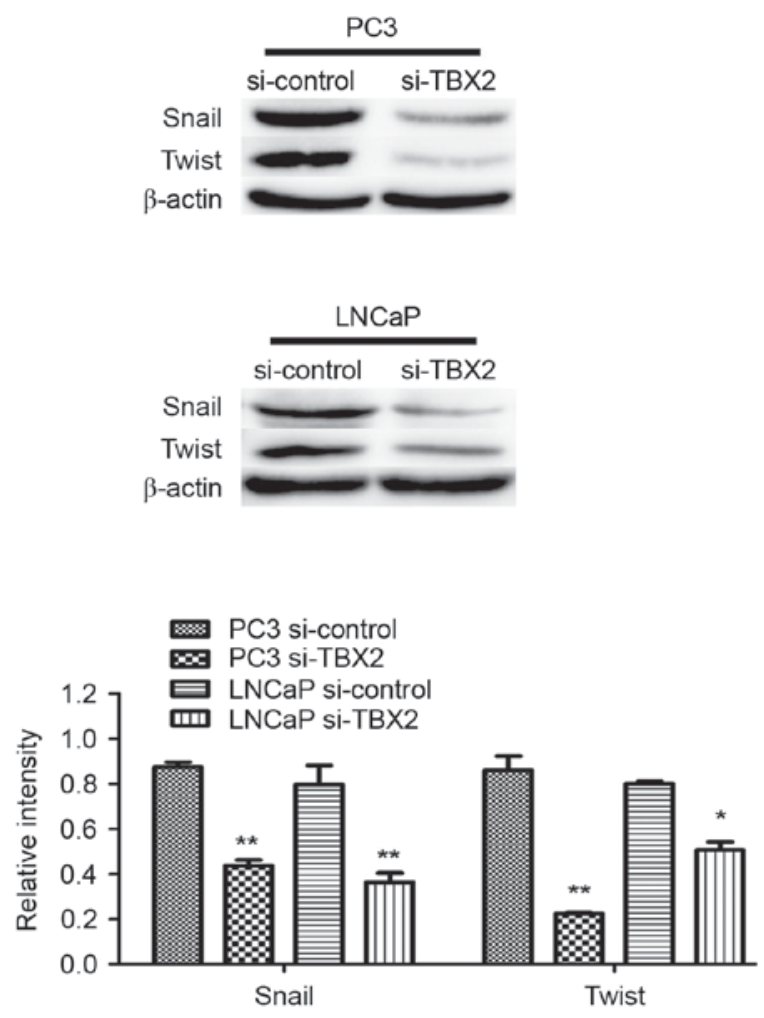

Figure 4. Silencing of TBX2 inhibits expression of Snail and Twist in PC3 and LNCaP cells. The protein level of EMT related transcription factors Snail and Twist were determined by Western blotting analysis. All data were shown as mean \pm SD of triplicates. ${ }^{* *} \mathrm{P}<0.01$.
A

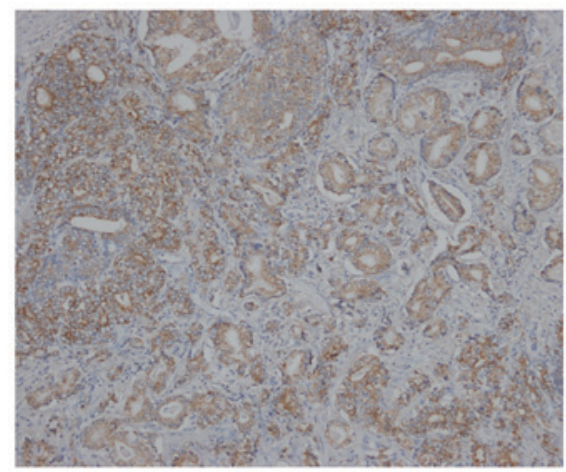

B

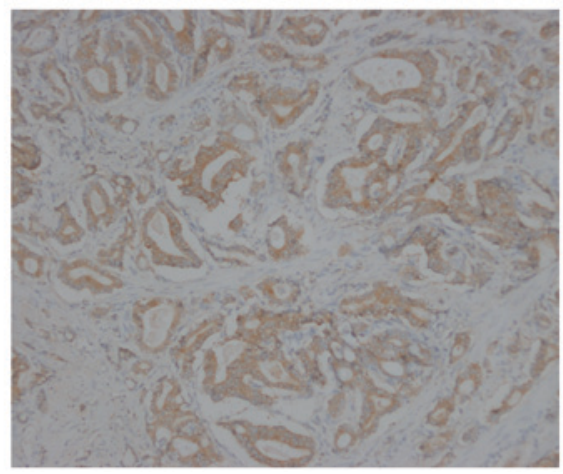

Figure 5. Representative immunohistochemical staining for TBX2 in prostate cancer. TBX2 was located in the cytoplasm of prostate cancer cells. The expression rates of TBX2 were $75.47 \%$ (40/53) in prostate cancer tissue, while $41.51 \%$ in tumor adjacent tissue $(\mathrm{P}<0.01)$. (A) Positive staining ' ++ ' for TBX2 in prostate cancer with well differentiation (x100). (B) Positive staining ' +++ ' for TBX2 in prostate cancer with poor differentiation (x100). 
Table I. The expression rates of TBX2 in prostatic cancer tissue and tumor adjacent tissue.

\begin{tabular}{lccccc}
\hline & \multicolumn{3}{c}{ TBX2 } & & \\
\cline { 3 - 4 } & Patients, n & + & - & $\chi^{2}$ & P-value \\
\hline Prostatic cancer tissue & 53 & 40 & 13 & 12.589 & $<0.01$ \\
Tumor adjacent tissue & 53 & 22 & 31 & & \\
\hline
\end{tabular}

TBX-2, T-Box-2; n, number of patients.

guide embryonic and tissue axial development, and play an inhibitory role in the process of regulation of development. Besides, the recent researches have explained TBX2 can be involved in cell cycle regulation, cell apoptosis and aggressive tumor growth through a variety of signaling pathways, as well as participate in EMT. Firstly, TGF- $\beta 1$ pathway has the ability to inhibit the proliferation of epithelial cells but promote their migration. Baldwin et al (6) have demonstrated that the TGF- $\beta 1$-mediated growth arrest could be bypassed by TBX2 overexpression. While the following study has shown that the downregulation of TBX2 through the direct binding of TBX3 to a half T-element in the TBX2 promoter, or the de-repression of the TBX2 target gene, p21, activates TGF- $\beta 1$ signaling pathway to exert its anti-proliferative effects (7). Furthermore,

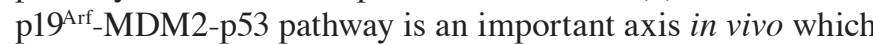
is associated with cell senescence. TBX2 represses transcription from the Arf tumor suppressor promoter, which decreases p53 activity by dampening the ability of $\mathrm{p} 19^{\mathrm{Arf}}(8)$, and consequently bypasses the normal senescence default mechanisms to restrain tumor cell apoptosis (9). In addition, E-cadherin, as a tumor suppressor, whose loss is implicated in EMT and metastatic tumor progression, is also a direct TBX2 target gene. The previous study has confirmed that RNAi-mediated silencing of TBX2 in two kinds of aggressive human breast carcinoma cell lines lead to re-expression of E-cadherin, and the concomitant loss of mesenchymal N-cadherin, Vimentin, and Fibronectin expression, which accordingly inhibit tumor cell migration, invasion, and EMT processes (2).

Based on the above data about its effects on cell migration, invasion and apoptosis in various cancers, we assumed that TBX2 gene also had the effect on cell migration, invasion and apoptosis in prostate cancer. Prior to this there were no researches about the TBX2 gene in prostate cancer including $\mathrm{PC} 3$ and $\mathrm{LNCaP}$ cells. Therefore, in order to determine the expression of TBX2 gene in PC3 and LNCaP cells, TBX2 siRNA and negative control siRNA were transfected into two types of prostate cancer cell lines respectively. Western blot analysis showed that the protein level of TBX2 was obviously repressed in TBX2 siRNA group, which demonstrated that the expression of TBX2 was effectively inhibited in cells transfected with TBX2 siRNA in PC3 and LNCaP cells. However, then we used the immunostaining to detect the expression of TBX2 in prostate cancer tissue and tumor adjacent tissue. The results showed that the expression rates of TBX2 in prostate cancer tissue were markedly higher than these in tumor adjacent tissue. Furthermore, TBX2 expression was correlated with clinical stage and pathological grade. In other words, the TBX2 expression was higher than in patients with poor differentiation or high clinical stage. The positive expression rates of TBX2 increased with the decrease of Gleason Score. On one hand, this might be due to the inhibition of p19 $9^{\text {Arf }}$ by TBX2 expression blocked cell senescence (10). On the other hand, the interference of PRb transcription via the expression of TBX2 might be influence on the normal operation of cell cycle and differentiation (11). Clinical staging is one of the important indexes, which could judge the prognosis of cancer sufferers. The patients with high clinical stage has greater probability of distant metastasis and poor prognosis. Our studies revealed that the activation of TBX2 gene might occur in the terminal stage of prostate cancer, which made it easier for prostate cancer to develop in the distance and increased the ability of invasion to progress toward a more malignant direction. Therefore, TBX2 gene has the remarkable characteristics of oncogene and is closely related to tumor invasion, which provides a new key factor for the prognosis of prostate cancer.

Then we preliminarily conducted the cell proliferation assay by CCK- 8 in order to test the cell proliferation among experimental interference (TBX2 siRNA) group and negative control group in the first place. The assay revealed that silencing of TBX2 led to reducing the ability of prostate cancer cell proliferation. Simultaneously, in order to determine the effect of downregulation of TBX2 gene for prostate cancer cell apoptosis, we also performed cell apoptosis assay by flow cytometry. The result showed that silencing TBX2 expression heightened apoptosis rate in both two kinds of prostate cancer cells. Next, we checked potential target genes involved in apoptosis of tumor cells in order to interpret the above experimental results and regulatory mechanisms of apoptosis caused by knockdown of TBX2 expression. We revealed that Bcl-2, MDM2 and p-Akt were downregulated, while Bax, p53 were upregulated. Bcl-2 family includes anti-apoptotic proteins such as $\mathrm{Bcl}-2, \mathrm{Bcl}-\mathrm{XL}$, and pro-apoptotic proteins such as Bax, Bad, Bid. The Bcl-2 family regulates apoptosis through Bcl-2 as an inhibitor or Bax as an activator $(12,13)$. In previous studies, we have confirmed Bcl-2 and Bax, whose activity is responsible for cell apoptosis (14), is dysregulated in many cancers (15). In our research, we got similar results that TBX2 siRNA might regulate $\mathrm{Bcl}-2$ and $\mathrm{Bax}$ to reduce the ability of cell apoptosis in prostate cancer. Furthermore, as we know, PI3K/AKT signaling plays a critical function during cancer cell proliferation and cell survival as well as invasiveness, angiogenesis and metastasis $(16,17)$. Akt activation might be a crucial promoter, not only leading to regulate other gene expression, but inhibiting cancer cell senescence and improving its growth. Phosphorylation of Akt and inhibition of androgen receptor via activation of Akt block the apoptosis of androgen dependent prostate cancer cells (18). Conditional activation of Akt has also been proved to promote the occurrence of androgen independent prostate cancer (19). MDM2, which participates in an auto-regulatory loop with p53, has been shown to function as a ubiquitin ligase that targets p53 for degradation. However, the activation and stabilization of p53 have also influence on function of MDM2, where any deletions in the pathways are determined to affect MDM2 expression in the cancer cells (20). Our results revealed that 
Table II. Clinicopathological parameters for TBX2 in prostatic cancer.

\begin{tabular}{|c|c|c|c|c|c|}
\hline \multirow[b]{2}{*}{ Clinicopathologic parameters } & \multirow[b]{2}{*}{ Patients, $\mathrm{n}$} & \multicolumn{2}{|c|}{$\mathrm{TBX} 2$} & \multirow[b]{2}{*}{$\chi^{2}$} & \multirow[b]{2}{*}{ P-value } \\
\hline & & + & - & & \\
\hline Age, years & & & & & 1.000 \\
\hline$<67$ & 18 & 14 & 4 & 0.003 & \\
\hline$\geq 67$ & 35 & 26 & 9 & & \\
\hline Clinical stage (TMN) & & & & & 0.025 \\
\hline $\mathrm{I}, \mathrm{II}$ & 33 & 21 & 12 & 5.031 & \\
\hline III, IV & 20 & 19 & 1 & & \\
\hline Pathological grade & & & & & $0.047^{\mathrm{a}}$ \\
\hline Well and moderate & 42 & 29 & 13 & & \\
\hline Poor & 11 & 11 & 0 & & \\
\hline PSA (ng/ml) & & & & & 0.331 \\
\hline$<10$ & 13 & 8 & 5 & 0.947 & \\
\hline$\geq 10$ & 40 & 32 & 8 & & \\
\hline
\end{tabular}

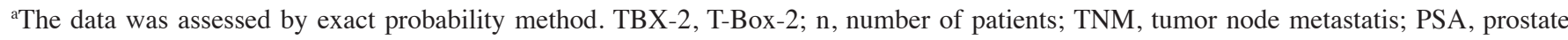
specific antigen.

TBX2 might be a contributor, which directly regulated phosphorylation of Akt. On one hand, low expression of p-Akt by silencing of TBX2 gene inhibited MDM2 activation, which sequentially enhanced the ability of $\mathrm{p} 53$, and then promoted cancer cell senescence. On the other hand, we showed that knockdown of TBX2 gene initiated cell senescence mechanism and inhibited cell progression through inhibition of phosphorylation of $\mathrm{Akt}$, whose activity directly promoted Bax expression.

The epithelial-mesenchymal transition is major mechanism that is related to cancer cell invasion and metastasis. It has reported that when the EMT process occurs, the phenotype of epithelial cells is transformed into the phenotype of mesenchymal cells, which weakens the ability of cell-cell adhesion, and then invades adjacent tissues to form metastases (21). It is marked by decreased expression of epithelial marker such as E-cadherin, while increased expression of mesenchymal markers such as $\mathrm{N}$-cadherin, Vimentin and Fibronectin. Wang et al (2) has clarified that TBX2 was related to invasion, migration and regulation of the progression of EMT in the development of malignant breast epithelial cells. Based on above results, we designed a similar experiment firstly in prostate cancer PC3 and LNCaP cells, and obtained similar results. We revealed that silencing of TBX2 increased E-cadherin expression and decreased N-cadherin, Vimentin and Fibronectin. In the meanwhile, cell migration and invasion assay also revealed that the number of cells of migration and invasion in the TBX2 siRNA group were significantly lower than that in the negative control group respectively. Our study indicated that TBX2 might be a target gene that directly regulated the expression of E-cadherin, and TBX2 knockdown was involved in the progression of EMT and reversed it through increasing E-cadherin expression.

In the increasing studies, we tried to determine whether TBX2 knockdown had any effect on expression of Snail and
Twist, which both played a crucial role in EMT. Snail, which is essentially a transcription repressor, is a major regulatory protein in EMT. By combining with the E-Box element located at E-cadherin promoter, Snail can directly inhibit the expression of E-cadherin, and then EMT can be induced to enhance cancer invasion and metastasis (22). Twist has been described as an oncogene due to its anti-apoptotic effects, but in recent studies, it has reported that Twist is highly expressed in various epithelial, mesenchymal and hematological malignant tumors, and is closely related to cell differentiation, lymph node metastasis and poor prognosis $(23,24)$. Twist has a similar mechanism of action with Snail. The deletion of Twist can lead to the loss of stem cells in tumor cells, which significantly inhibits the ability to initiate tumor (23). Consistent with these findings, we found that the protein expression of Snail and Twist markedly decreased in TBX2 siRNA group, which suggested that silencing of TBX2 might interfere with Snail/Twist signaling pathway. After TBX2 expression was knocked down in TBX2 siRNA group, Snail and Twist might have been unable to combine with the E-Box element of E-cadherin promoter, making it unable to initiate EMT, or even reverse EMT.

Neoangiogenesis, the formation of new blood vessels, is stimulated through growth factors, such as vascular endothelial growth factor, VEGF. These factors cause endothelial cells into the tumor tissue, where they form new capillaries to support tumor progression (25). In recent studies, we found that the function of VEGF might not only be limited to angiogenesis, but also play an important role in the survival, proliferation and migration of tumor cells (26). Therefore, we used western blot analysis to measure the VEGF expression level both in TBX2 siRNA group and negative control group. The result showed that the expression of VEGF was downregulated via silencing of TBX2 in TBX2 siRNA group, which indicated that downregulation of VEGF expression inhibited the formation of new blood vessels, and then prevented tumor progression. 
Taken together, our assays revealed that TBX2 gene may be a promoter of malignant tumor through induction of EMT process and inhibition of cell senescence. Specifically, in our studies downregulation of TBX2 suppressed the migration, invasion, proliferation, as well as EMT process, while promoting the apoptosis in PC3 and LNCaP cells. Meanwhile, according to the high expression of TBX2 in prostate cancer, TBX2 can help improve the accuracy in the clinical diagnosis of prostate cancer. The present study firstly demonstrated the effect of TBX2 gene in the prostate cancer, and based on our results, TBX2 might be a potential beneficial therapeutic target for prostate cancer treatment.

\section{References}

1. D'Costa ZC, Higgins C, Ong CW, Irwin GW, Boyle D, McArt DG, McCloskey K, Buckley NE, Crawford NT, Thiagarajan L, et al: TBX2 represses CST6 resulting in uncontrolled legumain activity to sustain breast cancer proliferation: A novel cancer-selective target pathway with therapeutic opportunities. Oncotarget 5: 1609-1620, 2014.

2. Wang B, Lindley LE, Fernandez-Vega V, Rieger ME, Sims AH and Briegel KJ: The T box transcription factor TBX2 promotes epithelial-mesenchymal transition and invasion of normal and malignant breast epithelial cells. PLoS One 7: e41355, 2012.

3. Han Y, Tu WW, Wen YG, Yan DW, Qiu GQ, Peng ZH and Zhou CZ: Increased expression of TBX2 is a novel independent prognostic biomarker of a worse outcome in colorectal cancer patients after curative surgery and a potential therapeutic target. Med Oncol 30: 688, 2013.

4. Vance KW, Carreira S, Brosch G and Goding CR: Tbx2 is overexpressed and plays an important role in maintaining proliferation and suppression of senescence in melanomas. Cancer Res 65: 2260-2268, 2005 .

5. Liu WK, Jiang XY and Zhang ZX: Expression of PSCA, PIWIL1, and TBX2 in endometrial adenocarcinoma. Onkologie 33: 241-245, 2010

6. Baldwin RL, Tran H and Karlan BY: Loss of c-myc repression coincides with ovarian cancer resistance to transforming growth factor beta growth arrest independent of transforming growth factor beta/Smad signaling. Cancer Res 63: 1413-1419, 2003.

7. Li J, Ballim D, Rodriguez M, Cui R, Goding CR, Teng H and Prince S: The anti-proliferative function of the TGF- $\beta 1$ signaling pathway involves the repression of the oncogenic TBX2 by its homologue TBX3. J Biol Chem 289: 35633-35643, 2014.

8. Jerome-Majewska LA, Jenkins GP, Ernstoff E, Zindy F, Sherr CJ and Papaioannou VE: Tbx3, the ulnar-mammary syndrome gene, and Tbx 2 interact in mammary gland development through a p19Arf/p53-independent pathway. Dev Dyn 234: 922-933, 2005 .

9. Redmond KL, Crawford NT, Farmer H, D'Costa ZC, O'Brien GJ, Buckley NE, Kennedy RD, Johnston PG, Harkin DP and Mullan PB: T-box 2 represses NDRG1 through an EGR1-dependent mechanism to drive the proliferation of breast cancer cells. Oncogene 29: 3252-3262, 2010.
10. Rowley M, Grothey E and Couch FJ: The role of Tbx2 and Tbx3 in mammary development and tumorigenesis. J Mammary Gland Biol Neoplasia 9: 109-118, 2004.

11. Vance KW, Shaw HM, Rodriguez M, Ott S and Goding CR: The retinoblastoma protein modulates Tbx2 functional specificity. Mol Biol Cell 21: 2770-2779, 2010.

12. Balogová L, Maslaňáková M, Dzurová L, Miškovský $\mathrm{P}$ and Stroffeková K: Bcl-2 proapoptotic proteins distribution in U-87 MG glioma cells before and after hypericin photodynamic action. Gen Physiol Biophys 32: 179-187, 2013.

13. Hojabrpour P, Waissbluth I, Ghaffari M, Cox ME and Duronio V: CaMKII- $\gamma$ mediates phosphorylation of BAD at Ser170 to regulate cytokine-dependent survival and proliferation. Biochem J 442: 139-149, 2012

14. Chao DT and Korsmeyer SJ: BCL-2 family: Regulators of cell death. Annu Rev Immunol 16: 395-419, 1998.

15. Adams JM and Cory S: The Bcl-2 apoptotic switch in cancer development and therapy. Oncogene 26: 1324-1337, 2007.

16. Courtney KD, Corcoran RB and Engelman JA: The PI3K pathway as drug target in human cancer. J Clin Oncol 28: 1075-1083, 2010.

17. Chang F, Lee JT, Navolanic PM, Steelman LS, Shelton JG, Blalock WL, Franklin RA and McCubrey JA: Involvement of PI3K/Akt pathway in cell cycle progression, apoptosis, and neoplastic transformation: A target for cancer chemotherapy. Leukemia 17: 590-603, 2003.

18. Vivanco I and Sawyers CL: The phosphatidylinositol 3-Kinase AKT pathway in human cancer. Nat Rev Cancer 2: 489-501, 2002.

19. Bertram J, Peacock JW, Tan C, Mui AL, Chung SW, Gleave ME, Dedhar S, Cox ME and Ong CJ: Inhibition of the phosphatidylinositol 3'-kinase pathway promotes autocrine Fas-induced death of phosphatase and tensin homologue-deficient prostate cancer cells. Cancer Res 66: 4781-4788, 2006.

20. Tan XL, Guo L and Wang GH: Polyporus umbellatus inhibited tumor cell proliferation and promoted tumor cell apoptosis by downregulating AKT in breast cancer. Biomed Pharmacother 83: 526-535, 2016.

21. Radisky DC and LaBarge MA: Epithelial-mesenchymal transition and the stem cell phenotype. Cell Stem Cell 2: 511-512, 2008.

22. Bonavida B and Baritaki S: Inhibition of Epithelial-to-Mesen chymal Transition (EMT) in cancer by nitric oxide: Pivotal roles of nitrosylation of NF- $\kappa$ B, YY1 and Snail. For Immunopathol Dis Therap 3: 125-133, 2012.

23. Yang J, Mani SA, Donaher JL, Ramaswamy S, Itzykson RA, Come C, Savagner P, Gitelman I, Richardson A and Weinberg RA: Twist, a master regulator of morphogenesis, plays an essential role in tumor metastasis. Cell 117: 927-939, 2004.

24. Kang Y and Massagué J: Epithelial-mesenchymal transitions: Twist in development and metastasis. Cell 118: 277-279, 2004.

25. Ferrara N: VEGF as a therapeutic target in cancer. Oncology 69 Suppl 3: 11-16, 2005.

26. Kowanetz $\mathrm{M}$ and Ferrara N: Vascular endothelial growth factor signaling pathways: Therapeutic perspective. Clin Cancer Res 12: 5018-5022, 2006. 